\title{
Research on the Teaching Platform of Criminal Image Technology Based on Virtual Reality \\ Feng $\mathrm{Xu}^{*}$
}

Department of Forensic Science \& Technology China Criminal Police University, China

* Corresponding author: Feng Xu, associate professor, doctor, xufeng_ccpc@hotmail.com

\begin{abstract}
The scene simulation is an important part of virtual reality system, it provides powerful methods support to the research of forensic science. In this paper, we introduce the connotation and characteristic of the virtual teaching, construct the teaching platform of criminal image technology and discuss the key problems of the function and model construction of virtual teaching platform in view of the actual situation that it is difficult to disassemble the equipment, establish simulation scene and the teaching content strong abstractness in the course of the criminal image technology teaching. The results show that it can better meet the diversity, richness, comprehensiveness and visualization requirements of teaching content, stimulate students to learn to drive, mobilize the initiative and enthusiasm of learning, through the construction of open, interactive, targeted and highly effective virtual platform.
\end{abstract}

Key words: visual simulation, virtual teaching, criminal image technology, simulated field

\section{Introduction}

With the implementation of strengthening police by technology, the forensic science technology has been placed in an important position. The criminal image is an important part of forensic science technology and one of the indispensable technical means to fight with all kinds of crimes ${ }^{1-4}$. In the process of handling cases, we must scientifically and legally obtain the evidence, examine the evidence, doubt the doubt of the evidence, and make full use of the information of the evidence in order to effectively expose and confirm the crime. At present, the teaching course of the criminal image technology still follows the traditional teaching mode, the introduction of image acquisition equipment mainly through the physical explanation and the scene photography scene needs to be set up and arranged in advance. With the complexity of image acquisition equipment and the improvement of packaging, it is difficult to explain the device components one by one. At the same time, it is difficult to completely construct the crime scene of the actual cases due to the limitations of space and resources, this greatly affects the effectiveness of classroom teaching ${ }^{5-7}$. Therefore, how to apply the latest information technology to the teaching of the course, focus on the training of creative thinking, activating thinking and developing cognitive ability, achieve the goal of teaching students to learn, give full play to the potential of the students and improve teaching efficiency become a key problem to be solved urgently.

\section{The connotation and characteristics of Virtual Teaching}

\subsection{The connotation of Virtual Teaching}

Virtual teaching is based on virtual reality technology, it is different from the traditional teaching. It stimulates people to immerse themselves in a variety of senses and interacts with 
the realistic environment at the same time, so as to achieve the purpose of rapid learning, real experience of life experience and ability improvement. Therefore, it can achieve the purpose of fast learning, real experience of life experience and ability improvement. According to the theory of system science, the system structure is the foundation of the whole system. The change of the structure leads to the change of the whole system. From the point of view of system science, the structure of virtual teaching and traditional teaching has changed, virtual reality technology plays an important role in virtual teaching system. According to the change of the relationship between the structural elements of the system, the interactions between the virtual teaching elements and the internal relations are more complex.

\subsection{The characteristics of Virtual Teaching}

\subsubsection{Multi-Sensory}

Multi-sensory refers to the general computer technology, in addition to the visual perception, as well as auditory perception, sensory perception, haptic perception, motion perception, and even taste perception, smell perception, etc.. The ideal virtual reality technology should have all people's perception function. Due to the limitations of the related technologies, especially the sensing technology, the current virtual reality technology has only a limited number of visual functions, such as vision, hearing, sense of force, touch, movement and so on.

\subsubsection{Immersion}

Immersion refers to the user feels the real extent of the protagonist in the simulation environment. The ideal simulation environment should make it difficult for users to distinguish between true and false, so that users can devote themselves to the $3 \mathrm{D}$ virtual environment created by computer. Everything in the environment seems to be true, it sounds true, even when it smells and tastes, and everything feels real, like in the real world.

\subsubsection{Interactivity}

Interactivity refers to the user's ability to operate in a simulated environment and the natural level of feedback from the environment (including real-time). For example, the user can use the hand to grab the virtual objects in the environment, then the hand holding the feeling of something, and you can feel the weight of the object, also caught in the view of objects can immediately move with the movement of hands.

\subsubsection{Imagination}

The virtual reality technology has broad imagination, it can broaden the scope of human cognition not only the real environment but also non-existent environment, even the impossible environment.

\section{Design goal of virtual teaching platform for criminal image technology}

The course of criminal image technology includes the image acquisition device for scene, digital image processing, scene image acquisition, physical evidence and so on. Teaching resources include multimedia material library, multimedia courseware, and teaching video and so on. The purpose of the course is to understand the situation of the crime scene for people who have not been to the scene by looking up the record from image acquisition 
equipment. To achieve this goal, the virtual reality technology is much more vivid than the traditional way. The user can directly access the simulation scene through the virtual teaching platform, which is built by the virtual engine, people can interact with each other, as if they were on the scene. The teaching platform has the following functions, as shown in Fig. 1.

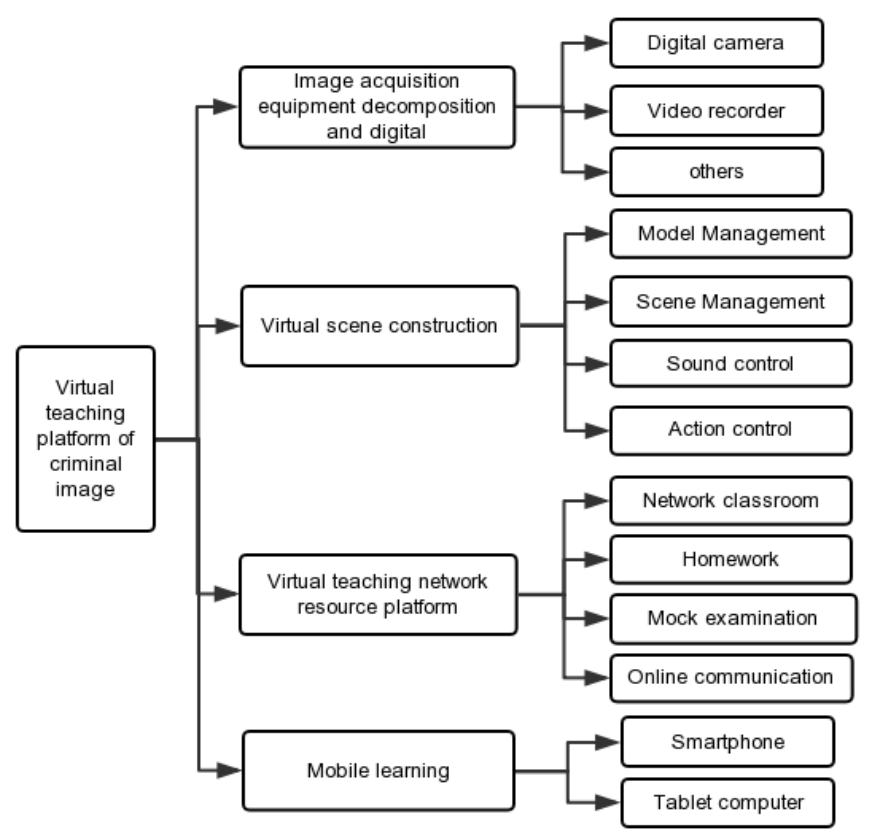

Fig. 1 -The structure of virtual teaching platform for criminal image technology

We can decompose and digitize it, and construct the 3D model database based on the virtual teaching environment through the research of the image acquisition equipment on the scene. Digital modelling of acquisition equipment for criminal image technology focuses on the functional requirements, structure and overall relationships of objects. It needs to meet the requirements of virtual environment acquisition equipment and the high information integration of the crime scene for the physical and dynamic attributes of the model.

The virtual scene building system is developed for the scene in the teaching. It can quickly realize the three-dimensional visual virtual reproduction of the case scene, meet the needs of classroom teaching, improve the efficiency of case detection and shorten the detection cycle. The virtual scene environment is a computer-generated three-dimensional virtual space, which is used to provide the mapping space for the objects in the real world and allow the participants to complete with the help of a certain interface, such as: DFA (Design For Assembly) modelling, path design, object position and attitude adjustment, blanking, view adjustment and view zoom, virtual simulation settings and other functions.

It can improve students' interest in learning knowledge of criminal science, arouse students' desire to explore knowledge and cultivate students 'autonomous learning ability by establishing the platform of network teaching resources. The platform is based on the professional knowledge learning of criminal image technology and integrates other related public security activities. The learning function breaks the traditional teaching structure and order in the breadth and depth. The platform can meet the needs of different levels of students (graduate students, in-service police training, undergraduate students and college students) on 
the basis of structured knowledge material of criminal image technology. Through the construction of collaborative platform, it can facilitate the cooperation and communication between teachers and students, teachers and public security, students and students.

Mobile learning is a new learning form, which can obtain educational information, educational resources and educational services from wireless mobile communication network technology and wireless mobile communication equipment. It is a digital activity through smart phones, tablet PCs and other intelligent terminals. In a sense, it is a supplementary extension of digital learning, which has the characteristics of mobility, high efficiency, universality, interactivity, sharing and personalization. Compared with digital teaching, mobile teaching not only has the characteristics of digitalization, multimedia, network and intelligence, but also has its unique advantages. Learners are no longer confined in front of the computer, you can short-flat-fast-learn anytime and anywhere. According to the specific circumstances of criminal image course, we construct the teaching methods with distinct characteristics from the five aspects of teaching thought, teaching objectives, mobile teaching resources, teachers' leading activities and the main activities of students, the application model.

\section{Key technologies of virtual teaching platform for criminal image}

Virtual reality system is an extremely complex system which integrates all kinds of advanced hardware and software technology, it must have the characteristics of flexibility, portability and real-time interaction. UDK is a software development kit and developed by Epic which can create simultaneous 3D model and be extendible. It provides a software access to simultaneous application of graph and high fidelity and multi-channel. We chooses UDK as the virtual engine because of its advantages such as real-time simulation, visual development, short development cycle, flexible application and powerful functions.

\subsection{Model Management}

In virtual teaching, the actual scene model is the key factor. UnrealED can not only generate static model, such as cube, cone, sphere, stairs, but also support mainstream 3D static model from 3DMAX or MAYA. The common models can be obtained from network; the special evidence in crime scene can be generated with ASE format by three-dimensional modeling software and be imported to UDK, as shown in Fig. 2. Digital process of criminal image acquisition equipment parameterized design using 3DMAX software after dismantling the parts.

\subsection{Scene Management}

In the system of virtual reality, the core is the virtual scene, which would create a cultural environment and reflect the objective world in fact. According to the room structure of real life, we build villa, farm house, bank, hotel, bar, shop, playground, three-bedroom and so on, as shown in Fig. 3.

\subsection{Sound control}

The sound production and control is a very important link to the virtual reality simulation fidelity. The sound of virtual reality is not only the common stereo sound effect, but rather 
than the more complex 3D stereo sound technology. It is sound technologies through the head recording technology, which can highly realistic simulate the various physical characteristics of the real world. The various sound files of various sound files can be imported into UDK with Wav format and saved to UPK pack-age through sound editor. We can control space and attenuation radius attribute to get 3D sound with attenuation node, mixing node, loop node, modulator node, continuous modulator node, random node and oscillator node.

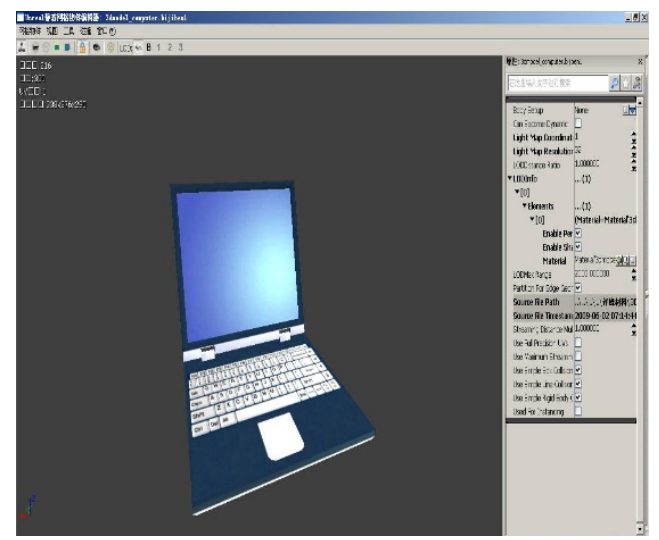

Fig. 2 -Static Model

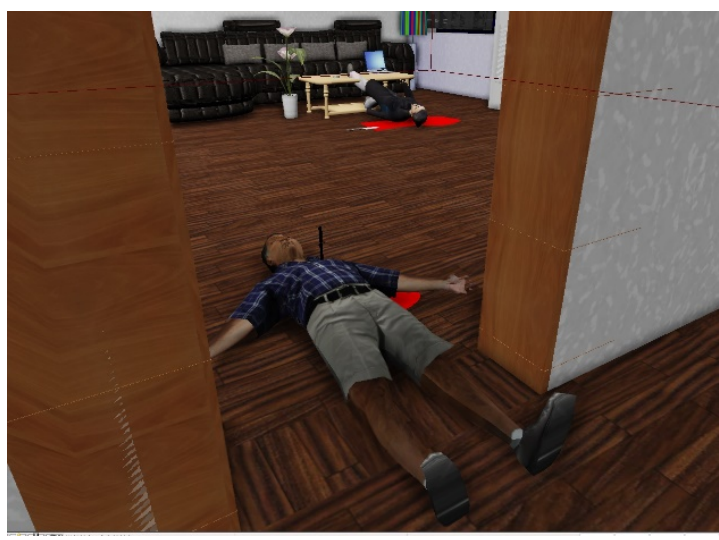

Fig. 3 -Virtual Scene

\subsection{Action control}

Virtual human is the digital representation of the various characteristics of human being in the virtual space, it plays an important role in improving the immersion of virtual reality application system. From the perspective of kinematics, the human body has more than 200 joints, the joints are connected by the skeleton and the skeleton is formed by the interaction of the joints. How to simulate the movement is one of the virtual human research which needs to be urgently solved. At present, the main methods of virtual human motion control are key frame method, kinematics and inverse kinematics, dynamics method and motion capture.

The animation system of Unreal Engine adds motion to render objects and entities. Animated objects are called Skeletal Meshes. Unlike Static Meshes (which can only be animated via Matinee), Skeletal Meshes have a set of bones to make up a skeleton. These bones are used to drive the motion of the object and placement of objects on a mesh. Animation trees are built using the AnimTree Editor, which is part of UnrealEd and can be seen in the Generic Browser in the same way as materials, meshes etc. We can modify animation and achieve satisfactory results with bone movement, skeleton controller and physical animation editor. We can simplify human animation, establish specific animation library, reconstruct the true crime scene and accomplish interactive function, which can promote the development of criminal technology, observe and analyse the crime scene from multi-angle, improve the efficiency and the accuracy of judgment.

\section{Conclusions}

On the premise of collecting, arranging, publishing, applying and evaluating the teaching resources of criminal imaging technology, virtual teaching platform of criminal image technology establishes the training mode of Applied Talents based on digital campus and virtual reality technology, which is one center (student-centered), three combination 
(combination of inside and outside class, combination of theory and Practice, combination of teaching and research), five-in-one (training in class, practicing outside the classroom, campus Practice, grassroots police practice, social case study). On the basis of the traditional teaching resources and forms, it can combine the research teaching, case teaching, mutual learning and innovative practice of modern teaching methods, realize the professional resources information by virtual interactive platform, reform the evaluation system and improve the teaching quality. In this paper, we explore the mode, method and law of new public security education system by virtual teaching. It has important theoretical and practical significance to the reform of related courses.

\section{Acknowledgements}

This research work is supported by the Ministry of public security technology research project under contract No. YJ20163066 and Education Science Planning Project of Liaoning Province under contract No. JG16DB482.

\section{References}

1. J. E.Deaton, C. Barba, T. Santarelli, L. Rosenzweig, V. Souders, et al, Virtual environment cultural training for operational readiness, J. Virtual Reality (2005), 8(3), 156-167.

2. M.Chen, Y.H. Sheng, Y.N. Wen, et al, Virtual Geographic Environments Oriented 3D Visualization System, Journal of System Simulation (2008), 20(19), 5105-5108.

3. J. R.Wilson, M. D. Cruz, Virtual and interactive environments for work for the future, International Journal of Human-Computer Studies (2006), 64(3), 158-169.

4. N. Hui, Research of the key technologies of autonomous virtual human, Tianjin: Tianjin University, 2007.

5. Z.Liu , J. Zhao , Psychology-based motivation model for intelligent virtual humans, Journal of System Simulation (2008), 20, 458-461.

6. L. Zhao, J.Y. Wang, B.J. Wang, Reduction of crime scene based on Ture Vision 3D technology, Journal of Chinese People's Public Security University (Science and Technology), 2008 (2), 85-88.

7. K.Zerrin, M. T.Nadia, Intelligent virtual humans with autonomy and personality: state-ofthe-art, Intelligent Decision Technologies, 2007(1), 3-15. 